\title{
GENERALIZATION OF AN INEQUALITY OF HEILBRONN AND ROHRBACH
}

\author{
F. A. BEHREND
}

Let $a_{1}, \cdots, a_{m}$ be positive integers and

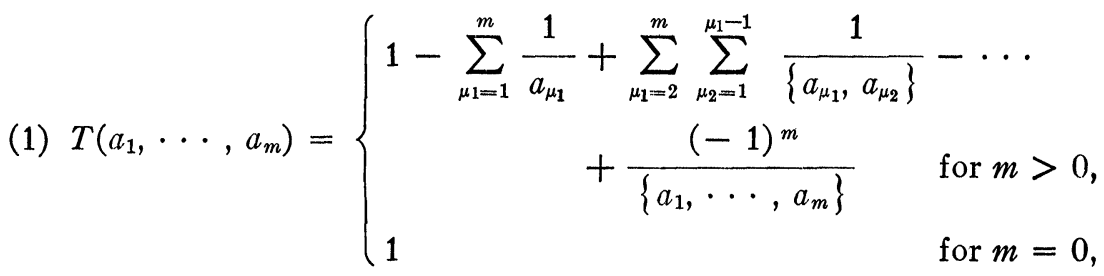

where $\left\{u_{1}, \cdots, u_{r}\right\}$ denotes the least common multiple of $u_{1}, \cdots, u_{r}$. H. A. Heilbronn ${ }^{1}$ and H. Rohrbach ${ }^{2}$ proved that

$$
\begin{aligned}
T\left(a_{1}, \cdots, a_{m}\right) & \geqq\left(1-\frac{1}{a_{1}}\right) \cdot \cdots \cdot\left(1-\frac{1}{a_{m}}\right) \\
& =T\left(a_{1}\right) \cdot \cdots \cdot T\left(a_{m}\right) .
\end{aligned}
$$

The object of this paper is to prove the following generalization of (2):

$$
\begin{aligned}
& T\left(a_{1}, \cdots, a_{m}, b_{1}, \cdots, b_{n}\right) \geqq T\left(a_{1}, \cdots, a_{m}\right) T\left(b_{1}, \cdots, b_{n}\right) \\
& \text { for } m \geqq 0, n \geqq 0 .
\end{aligned}
$$

$T\left(a_{1}, \cdots, a_{m}\right)$ may be interpreted as the density of the set $S$ of all positive integers not divisible by any $a_{\mu}$, that is,

$$
T\left(a_{1}, \cdots, a_{m}\right)=\lim _{z \rightarrow \infty} z^{-1} M(z),
$$

where $M(z)$ is the number of elements of $S$ not exceeding $z$.

For the proof of (3) we require the following lemma.

LEMMA. If $k \geqq 0, l \geqq 0$, and $\left(d, v_{\lambda}\right)=1$ for $\lambda=1, \cdots, l$, then

$$
\begin{aligned}
T\left(d u_{1}, \cdots, d u_{k}, v_{1}, \cdots, v_{l}\right) & \\
& =\frac{1}{d} T\left(u_{1}, \cdots, u_{k}, v_{1}, \cdots, v_{l}\right)+\left(1-\frac{1}{d}\right) T\left(v_{1}, \cdots, v_{l}\right) .
\end{aligned}
$$

Received by the editors March 14, 1947.

${ }^{1}$ On an inequality in the elementary theory of numbers, Proc. Cambridge Philos. Soc. vol. 33 (1937) pp. 207-209.

${ }^{2}$ Beweis einer zahlentheoretischen Ungleichung, J. Reine Angew. Math. vol. 177 (1937) pp. 193-196. 
Proof. $T\left(d u_{1}, \cdots, d u_{k}, v_{1}, \cdots, v_{l}\right)$ is the density of the set $S$ of all $x$ not divisible by any of $d u_{1}, \cdots, d u_{k}, v_{1}, \cdots, v_{l}$. We divide $S$ into two sets $S_{1}$ and $S_{2} . S_{1}$ contains all elements of $S$ which are divisible by $d$; they are of the form $x_{1}=d y$ subject to the condition that none of $d u_{1}, \cdots, d u_{k}, v_{1}, \cdots, v_{l}$ divides $d y$, which is equivalent to the condition that none of $u_{1}, \cdots, u_{k}, v_{1}, \cdots, v_{l}$ divides $y$; the density of the set of integers $y$ is thus $T\left(u_{1}, \cdots, u_{k}, v_{1}, \cdots, v_{l}\right)$ and the density of $S_{1}$ becomes $(1 / d) T\left(u_{1}, \cdots, u_{k}, v_{1}, \cdots, v_{l}\right), S_{2}$ contains all elements of $S$ which are not divisible by $d$; as $d u_{1}, \cdots, d u_{k}$ do not divide these elements, $S_{2}$ consists of all positive integers $x_{2}$ not divisible by any of $d, v_{1}, \cdots, v_{l}$, and its density is $T\left(d, v_{1}, \cdots, v_{l}\right)$. Thus we have

$$
\begin{aligned}
& T\left(d u_{1}, \cdots, d u_{k}, v_{1}, \cdots, v_{l}\right) \\
& \quad=\frac{1}{d} T\left(u_{1}, \cdots, u_{k}, v_{1}, \cdots, v_{l}\right)+T\left(d, v_{1}, \cdots, v_{l}\right) .
\end{aligned}
$$

Note that this proof still holds when one or both of $k, l=0$; for $k=0$, (4) reduces to

$$
T\left(v_{1}, \cdots, v_{l}\right)=\frac{1}{d} T\left(v_{1}, \cdots, v_{l}\right)+T\left(d, v_{1}, \cdots, v_{l}\right),
$$

whence

$$
T\left(d, v_{1}, \cdots, v_{l}\right)=\left(1-\frac{1}{d}\right) T\left(v_{1}, \cdots, v_{l}\right) .
$$

Substituting (6) in (4) we obtain the lemma.

PROOF OF (3): by induction with respect to $N=a_{1}+\cdots+a_{m}$ $+b_{1}+\cdots+b_{n}$. For $N=0, m=n=0$ and the three $T^{\prime}$ 's in (3) reduce to 1. Assume that (3) holds for $N^{\prime}<N$.

First case: Any two of $a_{1}, \cdots, a_{m}, b_{1}, \cdots, b_{l}$ relatively prime. In this case

$$
\begin{aligned}
T\left(a_{1}, \cdots, a_{m}, b_{1}, \cdots, b_{n}\right) & \\
& =\left(1-\frac{1}{a_{1}}\right) \cdot \cdots\left(1-\frac{1}{a_{m}}\right) \cdot\left(1-\frac{1}{b_{1}}\right) \cdot \cdots \cdot\left(1-\frac{1}{b_{n}}\right) \\
& =T\left(a_{1}, \cdots, a_{m}\right) T\left(b_{1}, \cdots, b_{n}\right) .
\end{aligned}
$$

Second case: $s$ exists such that $2 \leqq s \leqq m+n$ and (i) certain $s$ of the $a_{1}, \cdots, a_{m}, b_{1}, \cdots, b_{n}$ have a common divisor $d>1$, (ii) any $s+1$ of them have the greatest common divisor 1 (this condition being 
vacuous for $s=m+n)$. Rearranging the $a_{1}, \cdots, a_{m}, b_{1}, \cdots, b_{n}$ we may assume that $a_{1}, \cdots, a_{\mu}, b_{1}, \cdots, b_{\nu}$ have the common divisor $d>0$ where $\mu+\nu=s(\mu$ or $\nu$ may be 0$)$; then

$$
\begin{array}{lll}
a_{\rho}=d \bar{a}_{\rho} \text { for } \rho \leqq \mu ; & \left(a_{\rho}, d\right)=1 \text { for } \rho>\mu ; \\
b_{\sigma}=d \bar{b}_{\sigma} \text { for } \quad \sigma \leqq \nu ; & \left(b_{\sigma}, d\right)=1 \text { for } \sigma>\nu .
\end{array}
$$

By the lemma

$$
\begin{aligned}
T( & \left.a_{1}, \cdots, a_{m}\right) T\left(b_{1}, \cdots, b_{n}\right) \\
= & T\left(d \bar{a}_{1}, \cdots, d \bar{a}_{\mu}, a_{\mu+1}, \cdots, a_{m}\right) T\left(d \bar{b}_{1}, \cdots, d \bar{b}_{\nu}, b_{\nu+1}, \cdots, b_{n}\right) \\
= & \left(\frac{1}{d} T\left(\bar{a}_{1}, \cdots, \bar{a}_{\mu}, a_{\mu+1}, \cdots, a_{m}\right)+\left(1-\frac{1}{d}\right) T\left(a_{\mu+1}, \cdots, a_{m}\right)\right) \\
& \cdot\left(\frac{1}{d} T\left(\bar{b}_{1}, \cdots, \bar{b}_{\nu}, b_{\nu+1}, \cdots, b_{n}\right)+\left(1-\frac{1}{d}\right) T\left(b_{\nu+1}, \cdots, b_{n}\right)\right) \\
= & \frac{1}{d} T\left(\bar{a}_{1}, \cdots, \bar{a}_{\mu}, a_{\mu+1}, \cdots, a_{m}\right) T\left(\bar{b}_{1}, \cdots, \bar{b}_{\nu}, b_{\nu+1}, \cdots, b_{n}\right) \\
& +\left(1-\frac{1}{d}\right) T\left(a_{\mu+1}, \cdots, a_{m}\right) T\left(b_{\nu+1}, \cdots, b_{n}\right) \\
& -\frac{1}{d}\left(1-\frac{1}{d}\right)\left(T\left(a_{\mu+1}, \cdots, a_{m}\right)-T\left(\bar{a}_{1}, \cdots, \bar{a}_{\mu}, a_{\mu+1}, \cdots, a_{m}\right)\right) \\
& \cdot\left(T\left(b_{\nu+1}, \cdots, b_{n}\right)-T\left(\bar{b}_{1}, \cdots, b_{\nu}, b_{\nu+1}, \cdots, b_{n}\right)\right) .
\end{aligned}
$$

Observing that the first two terms may be estimated by the induction hypothesis and that the factors of the third term are not less than 0 , we get

$$
\begin{aligned}
& T\left(a_{1}, \cdots, a_{m}\right) T\left(b_{1}, \cdots, b_{n}\right) \\
& \leqq \frac{1}{d} T\left(\bar{a}_{1}, \cdots, \bar{a}_{\mu}, a_{\mu+1}, \cdots, a_{m}, b_{1}, \cdots, b_{\nu}, b_{\nu+1}, \cdots, b_{n}\right) \\
& \quad+\left(1-\frac{1}{d}\right) T\left(a_{\mu+1}, \cdots, a_{m}, b_{\nu+1}, \cdots, b_{n}\right) \\
&= T\left(a_{1}, \cdots, a_{m}, b_{1}, \cdots, b_{n}\right)
\end{aligned}
$$

by the lemma. Hence ( 3 ) is proved.

It is easy to decide when equality holds in (3). Equality will certainly hold if $\left(a_{\rho}, b_{\sigma}\right)=1$ for $\rho=1, \cdots, m, \sigma=1, \cdots, n$; this can be 
seen on the lines of the above proof, or, directly, by substituting the explicit value (1) of $T$ into (3) and observing that $\left\{a_{\rho_{1}}, \cdots, a_{p_{p}}\right\}$ $\cdot\left\{b_{\sigma_{1}}, \cdots, b_{\sigma_{q}}\right\}=\left\{a_{\rho_{1}}, \cdots, a_{\rho_{p}}, b_{\sigma_{1}}, \cdots, b_{\sigma_{q}}\right\}$. The converse is obviously not true as, for example, $T(2,4) T(3,6)=T(2,4,3,6)$; the reason is that, in this example, the numbers 4,6 are redundant; the example may be written simpler $T(2) T(3)=T(2,3)$. In general $u_{k}$ will be redundant in $T\left(u_{1}, \cdots, u_{k}\right)$ if it is a multiple of another $u_{\lambda}$. If redundant elements in $T\left(a_{1}, \cdots, a_{m}\right)$ and $T\left(b_{1}, \cdots, b_{n}\right)$ are removed, the converse of the above statement can be proved: If for some $\rho, \sigma\left(a_{\rho}, b_{\sigma}\right)>1$, inequality holds in (3). We may assume that $\left(a_{1}, b_{1}\right)>1$ and can apply $(7)$ with $\mu \geqq 1, \nu \geqq 1$. Now, if $u$ is not divisible by any of $v_{1}, \cdots, v_{l}$, then

$$
T\left(v_{1}, \cdots, v_{l}\right)>T\left(u, v_{1}, \cdots, v_{l}\right),
$$

for the set of positive integers not divisible by $v_{1}, \cdots, v_{l}$ contains the numbers $u\left(v_{1} \cdots v_{l} z+1\right), z=0,1,2, \cdots$, which possess a positive density and are not contained in the set of numbers not divisible by $u, v_{1}, \cdots, v_{l}$. As $a_{1}$, and hence $\bar{a}_{1}$, is not a multiple of any of $a_{\mu+1}, \cdots, a_{m}$ and $b_{1}$ not a multiple of any of $b_{\nu+1}, \cdots, b_{n}$, it follows that the factors of the last term of (7) are positive, and the inequality sign will hold in (8).

The University of Melbourne 